\title{
First record of Giardia assemblage D infection in farmed raccoon dogs (Nyctereutes procyonoides)
}

\author{
Piotr Solarczyk ${ }^{1}$, Anna C Majewska', Szymon Jędrzejewski', Marcin T Górecki², \\ Sławomir Nowicki ${ }^{3}$, Piotr Przysiecki ${ }^{4}$ \\ ${ }^{1}$ Department of Biology and Medical Parasitology, Medical Faculty I, Poznan University of Medical Sciences, Poland \\ 2 Department of Zoology, Institute of Zoology, Poznan University of Life Sciences, Poland \\ ${ }^{3}$ Department of Small Mammal Breeding and Animal Origin Materials, Poznan University of Life Sciences, Poland \\ ${ }^{4}$ Institute of Agriculture, Jan Amos Komenski State School of Higher Vocational Education in Leszno, Poland
}

Solarczyk P, Majewska AC, Jędrzejewski S, Górecki MT, Nowicki S, Przysiecki P. First record of Giardia assemblage D infection in farmed raccoon dogs (Nyctereutes procyonoides). Ann Agric Environ Med. 2016; 23(4): 696-698. doi: 10.5604/12321966.1226869

\section{Abstract}

The presence of Giardia genotypes was investigated in 18 raccoon dogs (Nyctereutes procyonoides) and 80 red foxes (Vulpes vulpes) on one farm. To demonstrate Giardia cysts, fresh and trichrome stained smears were microscopically screened. Two molecular markers were used for Giardia genotyping: a fragment of the beta-giardin gene and a fragment of the glutamate dehydrogenase gene. All faecal samples obtained from red foxes were negative. Giardia cysts were identified only in 2 of the 18 raccoon dogs. The result of genotyping and phylogenetic analysis showed that the $G$. duodenalis from both raccoon dogs belonged to the $D$ assemblage. This finding of a new animal reservoir of $G$. duodenalis canids-specific genotypes is important in order to eliminate the risk of infecting other animals bred for fur. Further molecular analyses of Giardia isolates in raccoon dogs are required. The present study represents the first contribution to knowledge of $G$. duodenalis genotypes in raccoon dogs.

\section{- Key words}

Giardia, molecular genotyping, raccoon dog

\section{INTRODUCTION}

Giardia duodenalis (syn. G. intestinalis, G. lamblia) is an intestinal protozoan parasite that infects humans and a wide range of animals including wild and domestic canids. This species exhibits great genetic diversity, and 8 major assemblages $(\mathrm{A}-\mathrm{H})$ have been defined [1]. These assemblages differ in host specificity: assemblages $\mathrm{A}$ and $\mathrm{B}$ are found in both humans and animals, whereas the rest of the assemblages are more host-adapted. There is increasing molecular evidence that canids may be infected with hostspecific genotypes (C and D) as well as zoonotic ones (A and $\mathrm{B})$; therefore, they may play a role as a potential source of Giardia infection impacting on humans and other Canidae. Although giardiasis in canids may be associated with various gastrointestinal abnormalities, most infected animals are asymptomatic [2].

The raccoon dog belongs to the Canidae family, and is native to Siberia and the Far East. The animals were introduced to Europe in the early 20th century, and now raccoon dogs are becoming more widespread throughout Europe and are considered as a potentially hazardous invasive species. The raccoon dog is the one of the animals used in the fur trade because of the quality of the pelt [3]. Diseases of animals are one of the indicators which provide information about farmed animal welfare. Although much research has been conducted on helminth fauna in raccoon dogs, there are limited data on their intestinal protozoan parasites $[4,5,6,7,8,9,10]$. To our knowledge, there is one report of Giardia infection in

Address for correspondence: Piotr Solarczyk, Department of Biology and Medical Parasitology, Medical Faculty I, Poznan University of Medical Sciences, Fredry 10 61-701 Poznań, Poland

E-mail: psolar@ump.edu.pl

Received: 26 August 2014; accepted: 17 September 2014 raccoon dogs; the parasite cysts were only microscopically observed in the feces of 3 out of 5 examined animals, and the Giardia isolates were not genotyped [7]. Therefore, the main aim of the present study was to determine the prevalence of Giardia genotypes in breeding raccoon dogs.

\section{MATERIALS AND METHOD}

Sample collection. In the presented study, a total of 18 fresh faecal samples were collected from farmed raccoon dogs on one small farm located in the west-central region of Poland. Since this farm specializes in breeding raccoon dogs and red foxes (Vulpes vulpes), faecal specimens sampled from 80 foxes were also examined. Each animal was kept in a separate cage. Fresh faecal specimens were taken from the ground below the bottom of the cage and placed into sterile plastic tubes, preserved in $2.5 \%$ potassium dichromate solution, transported to the laboratory in a cooler, and stored at $4{ }^{\circ} \mathrm{C}$ until they were analyzed. Two thin smears were made from each faecal specimen. One of them was examined as a fresh smear with a drop of Lugol's iodine solution and the second as a permanent trichrome stain. To demonstrate Giardia cysts and/or trophozoites, wet mounts and trichrome stained smears were microscopically screened using x 600 and x 1,000 magnification, respectively. In addition, part $(2 \mathrm{~g})$ of each faecal sample was concentrated using the $0.85 \mathrm{M}$ sucrose gradient centrifugation technique, with the final sediment being examined using a light microscope.

Molecular methods. Total genomic DNA was directly extracted from Giardia positive faecal samples. The FastDNA kit (BIO101, Vista, USA) was used for extraction of the Giardia DNA based on a protocol described earlier [11]. The eluted 
DNA was purified with the QIAquick PCR purification kit (Qiagen, Hilden, Germany) according to the kit instructions. Two molecular markers were used for Giardia genotyping: a $753 \mathrm{bp}$ fragment of the beta-giardin gene $(\mathrm{bg})$ was amplified using the G7 forward PCR primer and the G759 reverse primer, as previously described [12], and a $430 \mathrm{bp}$ fragment of the glutamate dehydrogenase gene $(g d h)$ was amplified using 2 forward primers (GDHeF, GDHiF) with one reverse primer (GDHiR) [13]. PCR amplification was performed under conditions previously reported $[12,13]$. Giardia DNA originating from cultured trophozoites of the Portland-1 reference strain was used as a positive control, whereas the negative control was a reaction mixture without the DNA template. Spike control was provided to rule out inhibitors. The PCR products were sequenced using the ABI Prism 3130 XL BigDye v3.1, Terminator Cycle Sequencing in both directions with the same set of primers.

Phylogenetic analysis. Both sequences were analyzed using Chromas and MEGA version 4.0 programmes. A phylogenetic tree was constructed by the neighbour-joining algorithm. Distance-based analyses were conducted using Kimura 2-parameter distance estimates using alignments obtained from ClustalW. Bootstrap proportions were calculated by analysis of the 1,000 replicates of the phylogenetic tree.

\section{RESULTS}

All faecal samples obtained from red foxes were negative for Giardia. Giardia cysts were identified only in 2 (NP1 and NP2) of the 18 raccoon dogs (11\%). Low infection intensity ranging from 12,000 - 13,000 cysts per gram of faeces was detected. Both Giardia infected animals were asymptomatic.

The partial $b g$ and $g d h$ sequences were successfully amplified in the NP1 and NP2 Giardia isolates. The sequences at both molecular markers from both NP1 and NP2 Giardia isolates were the same; it was also found that both Giardia isolates from the raccoon dogs belonged to the $\mathrm{D}$ assemblage. No double peaks in the chromatograms occurred in the tested loci.

The sequence of the $b g$ gene fragment of Giardia isolates obtained from the raccoon dogs (753 bp) shared $100 \%$ identity (D1-001, FJ009205) and 99\% similarity (A21, AY545647 and F42, HM061152) to the sequences from the reference isolates of $G$. duodenalis from dogs. The sequence of the $b g$ gene from both raccoon dog Giardia isolates differed only by one nucleotide substitution (1 SNP) from the A21 and F42 genotype D isolates. Comparison of the sequence of the $g d h$ gene fragment of the Giardia isolate from the raccoon dogs showed 100\% (NY24, JF958083) and 99\% (differed at this marker from 1 SNP to 4 SNPs) similarity to the sequences of this gene of the parasite isolated from dogs in various parts of the world (C29, EF507629; gidog2, DQ840535; NLDE3, AY827498; Dd4, AB569389). All mutations were synonymous. The sequences of the $b g$ and $g d h$ gene fragments from the G. duodenalis obtained from the raccoon dogs were deposited in GenBank (NCBI) under Accession Nos. HQ538708, HQ538710, HQ538709 and HQ538711, respectively. The phylogenetic analysis of the $g d h$ nucleotide sequences obtained from the NP1 and NP2 Giardia isolates placed them within clade D (99\% bootstrap support). Bootstrap analysis indicated strong statistical support for this grouping (Fig. 1).

\section{CONCLUSIONS}

Although G. duodenalis is the most prevalent parasitic intestinal protozoan to be found in wild, domestic and breeding Canidae in different regions of the world, this study has demonstrated a low rate of Giardia prevalence in raccoon dogs. This is probably due to the good hygienic conditions on the examined farm. Based on the molecular characteristics of Giardia isolates obtained from 2 raccoon dogs, it was determined that these animals were infected

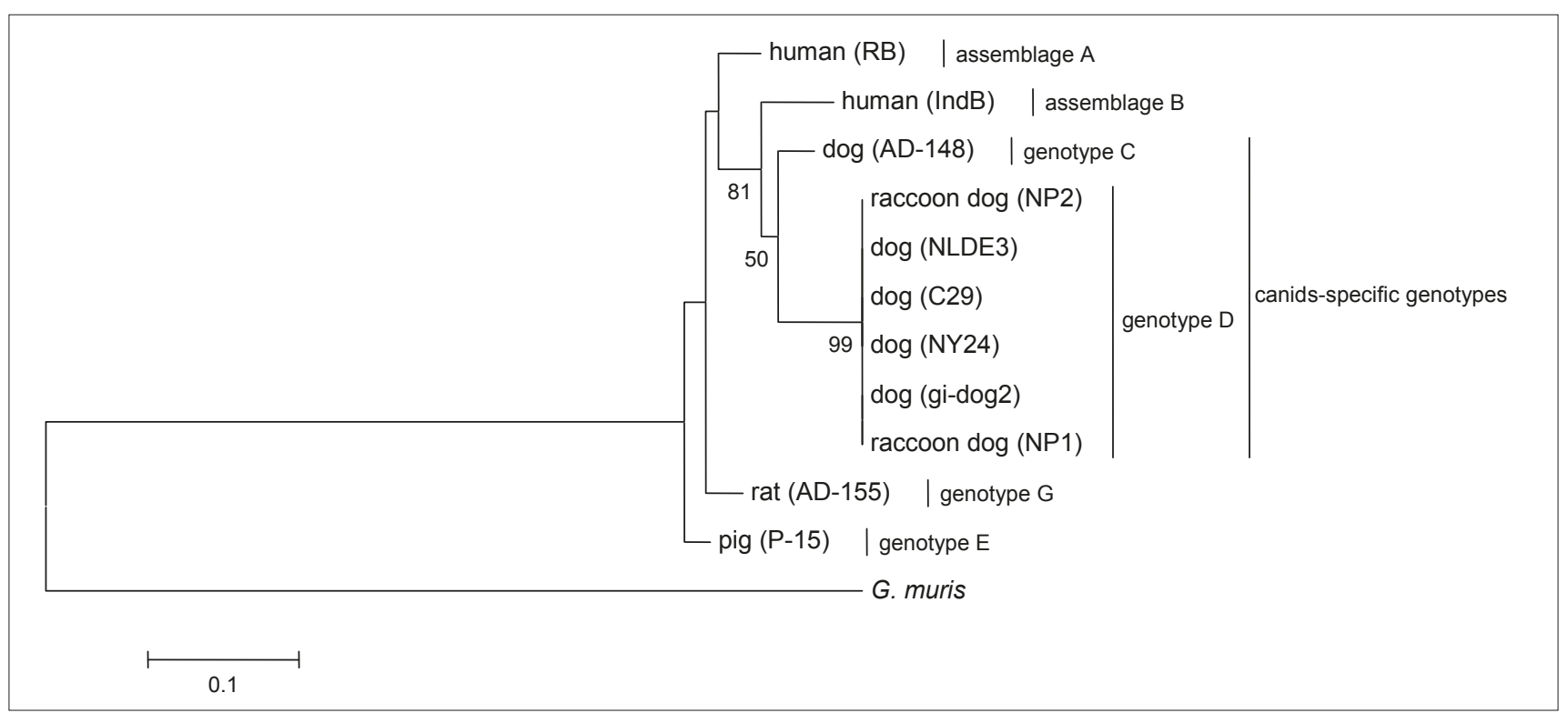

Figure 1. Phylogenetic relationship of 12 Giardia isolates inferred by the neighbour-joining analysis of glutamate dehydrogenase nucleotide sequences. Evolutionary distances computed using the Kimura 2-parameter method in the units of the number of base substitutions per site. Isolates from this study: NP1 and NP2 - from raccoon dogs. Reference isolates: RB (EF685702) and IndB (AB569386) represent assemblage A and assemblage B, respectively; AD-148 (U60982), NY24 (JF958083), C29 (EF507629), gi-dog2 (DQ840535), NLDE3 (AY827498) - reference dogs isolates; P-15-reference pig isolate (AY178741); AD-155 - reference rat isolate (AY178745). G. muris (AY258618) represents an outgroup. 
with the $\mathrm{D}$ genotype, which is adapted only to Canidae. However, recently it was found that 16 out of 17 Giardiapositive patients were infected with assemblage $\mathrm{C}$ which, like assemblage $\mathrm{D}$, is found in canids [14]. Therefore, it is also possible that Giardia assemblage D has zoonotic potential. Nevertheless, the finding of a new animal reservoir of $G$. duodenalis genotype $\mathrm{D}$, infectious to other animals bred for fur, is also important in order to eliminate the risk of infecting farmed raccoon dogs and red foxes. The rapid detection of Giardia infection and the treatment of fur breeding animals is necessary in order to provide good development conditions for these animals [15].

The presented study represents the first contribution to knowledge about the G. duodenalis genotypes in raccoon dogs. Further molecular analyses of Giardia isolates in raccoon dogs are required.

\section{Acknowledgments}

The study was supported by the University of Medical Sciences in Poznan, Poland (Grant No. 502-01-01123180033496). The authors also wish to thank Irena Robak for her excellent technical assistance.

\section{REFERENCES}

1. Ryan U, Cacciò SM. Zoonotic potential of Giardia. Int J Parasitol. 2013; 43: 943-956.

2. Barr SC, Bowman DD, Heller RL, Herb HN. Efficacy of albendazole against giardiases in dogs. Am J Vet Res. 1993; 54: 926-928.

3. Kowalska D, Bielański P. Finn raccoon what's next in Polish breeding? Wiad Zoot. 2011; 2: 43-51.

4. Thiess A, Schuster R, Nöckler K, Mix H. Helminth findings in indigenous raccoon dogs Nyctereutes procyonoides (Gray, 1843). Berl. Munch. Tierarztl. Wochenschr. 2001; 114: 273-276.
5. Machnicka-Rowinska B, Rocki B, Dziemian E, Kołodziej-Sobocińska M. Raccoon dog (Nyctereutes procyonoides) - the new host of Echinococcus multilocularis in Poland. Wiad Parazytol. 2002; 48: 65-68.

6. Shimalov VV, Shimalov VT. Helminth fauna of the raccoon dog (Nyctereutes procyonoides Gray, 1834) in Belorussian Polesie. Parasitol Res. 2002; 88: 944-945.

7. Shin SS, Cha DJ, Cho KO, Cho HS, Choi JO, Cho SH. Arthrostoma miyazakiense (Nematoda: Ancylostomatidae) infection in raccoon dogs of Korea and experimental transmission to dogs. Korean J Parasitol. 2007; 45: 121-128.

8. Shin EH, Park JH, Guk SM, Kim JL, Chai JY. Intestinal helminth infections in feral cats and a raccoon dog on Aphaedo Island, Shinangun, with a special note on Gymnophalloides seoi infection in cats. Korean J Parasitol. 2009; 47: 189-191.

9. Bruzinskaite-Schmidhalter R, Sarkunas M, Malakauskas A, Mathis A, Torgerson PR, Deplazes P. Helminths of red foxes (Vulpes vulpes) and raccoon dogs (Nyctereutes procyonoides) in Lithuania. Parasitology. 2012; 139: 120-127.

10. Al-Sabi MNS, Chriél M, Jensen TH, Enemark HL. Endoparasites of the raccoon dog (Nyctereutes procyonoides) and the red fox (Vulpes vulpes) in Denmark 2009-2012 - A comparative study. Int J Parasitol Parasites Wildl. 2013; 2: 144-151.

11. da Silva AJ, Bornay-Llinares FJ, Moura IN, Slemenda S, Tuttle JL, Pieniazek NJ. Fast and reliable extraction of protozoan parasite DNA from fecal specimens. Mol Diag. 1999; 4: 57-64.

12. Cacciò SM, De Giacomo M, Pozio E. Sequence analysis of the betagiardin gene and development of a polymerase chain reaction restriction fragment length polymorphism assay to genotype Giardia duodenalis cysts from human faecal samples. Int J Parasitol. 2002; 32: 1023-1030.

13. Read CM, Monis PT, Thompson RC. Discrimination of all genotypes of Giardia duodenalis at the glutamate dehydrogenase locus using PCR-RFLP. Infect Genet Evol. 2004; 4: 125-130.

14. Liu H, Shen Y, Yin J, Yuan Z, Jiang Y, Xu Y, Pan W, Hu Y, Cao J. Prevalence and genetic characterization of Cryptosporidium, Enterocytozoon, Giardia and Cyclospora in diarrheal outpatients in China. BMC Infect Dis. 2014; 14: 1- 6.

15. Anderson KA, Brooks AS, Morrison AL, Reid-Smith RJ, Martin SW, Benn DM, Peregrine AS. Impact of Giardia vaccination on asymptomatic Giardia infections in dogs at a research facility. Can Vet J. 2004; 45: 924-930. 